\title{
Mechanism of Lipid Mobilization by the Small Intestine after Transport Blockade
}

\author{
J. Halpern, P. Tso, and C. M. Mansbach II \\ Departments of Medicine and Physiology, The University of Tennessee, Memphis, Memphis, Tennessee 38163
}

\begin{abstract}
The nonionic detergent, Pluronic L-81 (L-81) has been shown to block the transport of intestinal mucosal triacylglycerol (TG) in chylomicrons. This results in large lipid masses within the enterocyte that are greater in diameter than chylomicrons. On removal of $\mathrm{L}-81$, mucosal TG is rapidly mobilized and appears in the lymph. We questioned whether the blocked TG requires partial or complete hydrolysis before its transport. Rats were infused intraduodenally with $\left[{ }^{3} \mathrm{H}\right]$ glyceryl, $\left[{ }^{14} \mathrm{C}\right]-$ oleoyl trioleate (TO) and $0.5 \mathrm{mg} \mathrm{L-81/h}$ for $8 \mathrm{~h}$, followed by $120 \mu \mathrm{mol} / \mathrm{h}$ linoleate for $18 \mathrm{~h}$. Mesenteric lymph was collected and analyzed for TG content and radioactivity. An HPLC method was developed to separate TG on the basis of its acyl group species. The assumed acyl group composition was confirmed by gas liquid chromatography analysis. TG lymphatic output was low for the first $8 \mathrm{~h}$ but increased to $52 \mu \mathrm{mol} / \mathrm{h}$ at the 11th $h$ of infusion ( $3 \mathrm{~h}$ after stopping $\mathrm{L}-81$ ). $38 \%$ of the infused TO was retained in the mucosa after the 8-h infusion. 95\% of mucosal TG was TO, $92 \%$ of the radioactivity was in TG, and $2.4 \%$ of the ${ }^{14} \mathrm{C}$ disintegrations per minute was in fatty acid. HPLC analysis of lymph at $6,10,12$, and $14.5 \mathrm{~h}$ of infusion showed a progressive rise in TG composed of one linoleate and two oleates, to $39 \%$; and in TG composed of two linoleates and one oleate to $20 \%$ at $14.5 \mathrm{~h}$ of infusion. On a mass basis, however, $80 \%$ of the TG acyl groups were oleate. ${ }^{3} \mathrm{H} /{ }^{14} \mathrm{C}$ ratios in the various TG acyl group species reflected the decrease in oleate. We conclude that first, unlike liver, most mucosal TG is not hydrolyzed before transport. The mechanism of how the large lipid masses present in mucosal cells after L-81 infusion are converted to the much smaller chylomicrons is unknown. Second, the concomitant infusion of linoleate did not impair lymph TG delivery after L-81 blockade.
\end{abstract}

\section{Introduction}

Pluronic L-81 (L-81) ${ }^{1}$ is a hydrophobic detergent made up of polyoxyethylene and polyoxypropylene copolymers. Its average molecular weight is 2,750 . Previous observations have

Address reprint requests to Dr. Charles M. Mansbach II, The University of Tennessee at Memphis, 951 Court Avenue, Room 555 Dobbs, Memphis, TN 38163.

Received for publication 31 August 1987 and in revised form 14 December 1987.

1. Abbreviations used in this paper: CM, chylomicrons; dpm, disintegrations per minute; L-81, Pluronic L-81; TG, triacylglycerol; TL, glyceryl trilineolate; TO, glyceryl trioleate.

\footnotetext{
J. Clin. Invest.

(C) The American Society for Clinical Investigation, Inc.

0021-9738/88/07/0074/08 \$2.00

Volume 82, July $1988,74-81$
}

demonstrated that $\mathrm{L}-81$ is a potent inhibitor of intestinal lipid transport (1-3). The mechanism involved is the inhibition of the formation and transport of intestinal chylomicrons (CM) but not of the smaller intestinal VLDL. Electron microscopic data suggests that L-81 causes an accumulation of triacylglycerol (TG) in vesiculated smooth endoplasmic reticulum and prevents the movement of lipid droplets into the Golgi complex (2). These lipid droplets, which accumulate as a result of L-81, have diameters varying between 2 and 5,000 $\mathrm{nm}$. As a consequence; they are 10-fold larger than CM. The Golgi apparatus appears normal in response to L-81, and VLDL-sized lipoproteins are seen being processed in the Golgi vacuoles. The lipoproteins present in the intercellular space of the normal animals are mostly CM, whereas only VLDL are present in the intercellular space of L-81-treated rats. VLDL secreted by the L-81-treated enterocytes thus is first processed by the Golgi apparatus before their release.

The inhibitory action of L-81 is both rapid (2) and reversible upon the cessation of L-81 infusion. This raises the question of how the lipid within the large droplets is reduced to CM-sized particles. Although there is information regarding the mobilization of accumulated TG in some organs, e.g., the liver (4), the mobilization of the lipid accumulated in the small intestine remains unknown. In the liver, lysosomal acid lipase has been shown to play an important role in this process (4). This involves initial hydrolysis of the TG accumulated in the liver followed by TG resynthesis in microsomes before its transport by the hepatocytes.

The aim of this study was to determine if TG, accumulated in the intestinal mucosa in response to L-81 infusion, also required hydrolysis before its transport out of the cells as TG in $\mathrm{CM}$. To answer this question, we first loaded the intestinal cell in the presence of L-81 with glyceryltrioleate (TO) whose glycerol moiety was labeled with ${ }^{3} \mathrm{H}$ and each oleoyl residue labeled with ${ }^{14} \mathrm{C}$. We then stopped the L-81/TO infusion and started an infusion of linoleate in an amount calculated to give the same quantity of fatty acid as was provided by the TO. Several questions could be answered during this unblocking period. First, was the labeled TG that appeared in the lymph mainly TO, or was it significantly mixed with linoleate? If it appeared as TO, then no hydrolysis was required before transport. Any admixture of linoleate would suggest hydrolysis with reesterification with the linoleate that was flooding the intestinal cell during the unblocking period. The extent to which the linoleate was present would indicate the degree of hydrolysis of the original TO. The glyceride glycerol originally esterified to TO could be identified because of its ${ }^{3} \mathrm{H}$ label. If the TO molecule were completely hydrolyzed, then it would be unlikely that the labeled glycerol would be reused for TG synthesis. The reasons for this have been given previously (5) and can be directly tested in this study by determining whether any glyceryl trilinoleate (TL) contains ${ }^{3} \mathrm{H}$.

The second question that could be answered is whether the administration of a transportable lipid would hinder the appearance of TG stored in the intestinal cell in response to L-81. 
The rate of appearance in the lymph of the stored radiolabeled TG would indicate whether the intestinal cell could mobilize this lipid with the same rapidity as when saline was used during the unblocking period. In this study it also had to process newly absorbed FFA from the lumen, which also required transport.

\section{Methods}

Animals. Male Sprague-Dawley rats (250-300 g) were fasted overnight before surgery. While under ether anesthesia, the rats main mesenteric lymph duct was cannulated with a clear vinyl tubing $(0.8-\mathrm{mm}$ o.d.) according to the method of Bollman et al. (6). To prevent clotting, the lymph cannula was primed with heparin sodium solution supplied by ICN Pharmaceuticals, Inc. (Cleveland, $\mathrm{OH}$ ). Silicone tubing (1.6-mm o.d., tipped inside with a $1.0-\mathrm{mm}$ o.d. clear vinyl tubing) was introduced $\sim 2 \mathrm{~cm}$ down the duodenum through the fundus of the stomach. The tubing was secured in the duodenum through a transmural suture and the fundal incision was closed by a purse-string suture. After surgery, the animals were infused via the duodenal tube at a rate of 3 $\mathrm{ml} / \mathrm{h}$ with a $5 \%$ dextrose saline solution $(145 \mathrm{mM} \mathrm{NaCl}, 4 \mathrm{mM} \mathrm{KCl}$, and $0.28 \mathrm{M}$ dextrose). The animals were allowed to recover for at least $36 \mathrm{~h}$ in restraining cages kept in a warm chamber $\left(\sim 30^{\circ} \mathrm{C}\right)$ before the lipid infusion.

Experimental plan. In the main series of experiments, three groups of lymph fistula rats were used (A, B, and C). On the day of the experiment, the three groups of lymph fistula rats were infused for $8 \mathrm{~h}$ ( $3 \mathrm{ml} / \mathrm{h}$ ) with a lipid emulsion containing $40 \mu \mathrm{mol}$ TO (labeled with 25 $\mathrm{nCi} / \mu \mathrm{mol}$ of $\left[{ }^{3} \mathrm{H}\right] \mathrm{glyceryl}$ trioleate and $2.5 \mathrm{nCi} / \mu \mathrm{mol}$ of glyceryl tri $\left[{ }^{14} \mathrm{C}\right]$ oleate), $8.8 \mu \mathrm{mol}$ of phosphatidylcholine, $57 \mu \mathrm{mol}$ sodium taurocholate, and $0.5 \mathrm{mg} \mathrm{L}-81$ in $3 \mathrm{ml}$ of PBS. The PBS (pH 6.4) contained $6.75 \mathrm{mM} \mathrm{Na}_{2} \mathrm{HPO}_{4}, 16.5 \mathrm{mM} \mathrm{NaH}_{2} \mathrm{PO}_{4}, 115 \mathrm{mM} \mathrm{NaCl}$, and 5 $\mathrm{mM} \mathrm{KCl}$. In group A, the animals were killed at the end of $8 \mathrm{~h}$ of this lipid infusion. The various gastrointestinal, luminal, and mucosal samples were analyzed for radioactive lipid content. In group B, after $8 \mathrm{~h}$ of TO plus L-81 infusion, the infusate was changed to a linoleate lipid emulsion containing $120 \mu \mathrm{mol} / \mathrm{h}$ linoleate, $8.8 \mu \mathrm{mol}$ egg phosphatidylcholine, and $57 \mu \mathrm{mol} / \mathrm{h}$ sodium taurocholate, which was continued at $3 \mathrm{ml} / \mathrm{h}$ for 20 additional hours. No L-81 was added to this infusate. In group $\mathrm{C}$, the 8-h infusion of the TO plus L-81 lipid emulsion was followed by the infusion of a $5 \%$ dextrose saline solution as described above.

Lymph from the three groups of rats was collected hourly into precooled tubes. Lymph collected $1 \mathrm{~h}$ before lipid infusion was analyzed as the fasting lymph. Aliquots of lymph samples were taken for both TG determination (7) and for the counting of ${ }^{3} \mathrm{H}$ - and ${ }^{14} \mathrm{C}$-radioactivity by scintillation spectrometry. For TG determination, the lipid was extracted by the method of Blankenhorn and Ahrens (8) before analysis.

In group A, rats were killed by ether anesthesia at the end of the TO plus L-81 infusion. The small intestine was removed, divided into quarters, and placed in $0.15 \mathrm{M} \mathrm{NaCl}$ at $4^{\circ} \mathrm{C}$. After removing the remaining mesentery, each segment was opened longitudinally and the mucosa cleaned by rinsing with $0.15 \mathrm{M} \mathrm{NaCl}$ and then blotted with a piece of tissue paper. The mucosa was scraped using a microscope slide. The mucosal scrapings were collected in beakers containing $5 \mathrm{ml}$ of $0.15 \mathrm{M} \mathrm{NaCl}$ and homogenized with 10 strokes in a 7-ml glass (Thomas Scientific, Philadelphia, PA) and Teflon homogenizer. An aliquot of the homogenate was taken for lipid extraction by the method of Folch et al. (9). A portion of the extract was taken for radioactivity determination. Another portion was separated into lipid classes by TLC using a solvent system of petroleum ether/diethyl ether/acetic acid (84:15:1, vol/vol). The spots representing the cholesteryl ester, TG, fatty acid, diacylglycerol, monoacylglycerol, and phospholipids were scraped into $20-\mathrm{ml}$ scintillation vials. After addition of $1 \mathrm{ml}$ ethanol and $9 \mathrm{ml}$ toluene-based scintillant, the radioactivity was determined.
Group $C$ was identical to group B except that the rats were infused with 5\% dextrose saline solution instead of the linoleate emulsion. This group of rats was studied as a control for group B rats and also to ensure that the rats in this study behaved similarly to those in our previous studies (3).

HPLC procedures. HPLC was performed using a system equipped with dual pumps and a variable wavelength detector (model 110; Beckman Instruments, Fullerton, CA). Separations were performed on a $4.6 \mathrm{~mm}$ i.d. $\times 25 \mathrm{~cm}$ HPLC column (ZORBAX ODS; DuPont Co., Diagnostic \& Bioresearch Systems, Wilmington, DE) whose particle size was $5 \mu \mathrm{m}$. This column was preceded by a guard column (ZORBAX ODS), $1.25 \mathrm{~cm}$ long. The flow rate was $1 \mathrm{ml} / \mathrm{min}$. Preliminary data using TL dissolved in l-propanol demonstrated an absorbance maximum at $210 \mathrm{~nm}$. This was chosen as the wavelength to monitor the column effluent. The appearance of lipids from the column was recorded (model BD 41 recorder; Kipp and Zonen, Delft, The Netherlands) and the eluent collected using a 2-min sampling time with the aid of a fraction collector (Retriever II; Isco, Inc., Lincoln, NE). Chromatograms were developed using $56 \%$ acetonitrile (UV grade; Burdick \& Jackson Laboratories, Inc., American Hospital Supply Corp., Muskegon, MI) and 44\% l-propanol (vol/vol) (HPLC grade; Burdick and Jackson Laboratories, Inc.) in an isocratic mode.

Using this chromatographic system, TL had a retention time of 18 min; glyceryl dilinoleate-monooleate, $24 \mathrm{~min}$; glyceryl monolinoleatedioleate, $31 \mathrm{~min}$; and TO, $36 \mathrm{~min}$. Proof of these structures was established by gas liquid chromatography (GLC), which is described below. Further proof was obtained by the chromatography of pure standards in the case of TL and TO, whose retention times were as predicted, and chromatographing glyceryl $\left[{ }^{14} \mathrm{C}\right]$ trioleate (New England Nuclear, Boston, MA), in which case all the radioactivity appeared between 37 and 39 min after injection.

Samples were prepared for HPLC in the case of lymph by extracting the lymph of its lipid content (9), evaporating the chloroform phase under $\mathrm{N}_{2}$, and dissolving the lipids in l-propanol. The extract was treated with fluorocil to remove phospholipids (10). Mucosal lipid extracts were first separated by TLC (11) to isolate the TG fraction. The TG was eluted from the silica gel thrice with $5 \mathrm{ml}$ chloroform. The chloroform was evaporated under $\mathrm{N}_{2}$ and the TG dissolved in l-propanol.

To determine the distribution of radioactivity among TGs of specific acyl group composition, $\sim 350 \mathrm{nmol}$ TG was chromatographed. Collection tubes representing the entire chromatogram were evaporated to dryness using a vortex evaporator (Haake Buchler Instruments, Inc., Saddle Brook, NJ). The dried lipids were washed into scintillation vials using the scintillation medium as described previously (12). Radioactivity was determined on a liquid scintillation analyzer (model 1500; Packard Instrument Co., Downers Grove, IL). This instrument was programmed with quench curves for both ${ }^{3} \mathrm{H}$ and ${ }^{14} \mathrm{C}$, which enabled the calculation of disintegrations per minute (dpm) from counts per minute for each radionuclide in the presence of the other.

To determine the fatty acid composition of TG species, the TG fractions isolated from the HPLC were evaporated under nitrogen. Methyl esters of the fatty acid were prepared by methylation with boron trifluoride-methanol (Supelco, Inc., Bellefonte, PA) in the absence of air according to the method of Morrison and Smith (13). The fatty acid methyl esters were separated and quantitated by GLC (Mini 2; Shimadzu Seisakusho Ltd., Kyoto, Japan) equipped with a chromatopak CR 1B. A 30-mm-long $\times 0.25$-mm-diam open tubule column with a $0.2-\mu \mathrm{m}$ stationary phase of SP-2330 was used for separation (Supelco, Inc.). The carrier gas was helium. The column temperature was $210^{\circ} \mathrm{C}$ for $18 \mathrm{~min}$, advancing at $2^{\circ} \mathrm{C} / \mathrm{min}$ for $8 \mathrm{~min}$. The injector/ detector temperature was $240^{\circ} \mathrm{C}$. Identification of individual fatty acids was based on known standards.

Radioactivity determination. Radioactivity was measured in a water-miscible scintillant (Aquasolv; Beckman Instruments) for aqueous solutions. The other samples used a toluene-based scintillant (12). Samples were counted for $10 \mathrm{~min}$ in a liquid scintillation spec- 
trometer (model 1500; Packard Instruments) in a dual label mode. Samples were corrected for quenching by reference to a series of ${ }^{3} \mathrm{H}$ and ${ }^{14} \mathrm{C}$ standards that had been progressively quenched.

Materials. Glyceryl tri $\left[l^{-14} \mathrm{C}\right]$ oleate were supplied by New England Nuclear. $\left[{ }^{3} \mathrm{H}\right]$ TO was purchased fróm Amersham Corp., Arlington Heights, IL. TO was purchased from Sigma Chemical Co. (St. Louis, MO) and was found to be $>99 \%$ pure. Egg phosphatidylcholine, sodium taurocholate, and linoleate were obtained from Sigma Chemical Co. and were used without further purification. L-81 surfactant was kindly donated by BASF Wyandotte Corp., Parsippany, NJ. All reagents or solvents used were of analytical grade.

Statistical analysis. Differences between groups were analyzed by the two-tailed $t$ test.

\section{Results}

Preliminary experiments. At the conclusion of the 8-h TO plus L-81 infusion, $70 \%$ of the ${ }^{3} \mathrm{H}$ and ${ }^{14} \mathrm{C}$ dpm remaining in the mucosa were found in the most proximal quarter of the intestine. The second quarter contained the remainder of the dpm thăt were still present. All the dpm thus were in the proximal half of the gut at the time of removal of L-81 from the infusate.

We next wished to observe the percentage of total infused lipid remaining in the mucosa at the conclusion of the TO $+\mathrm{L}-81$ infusion. At the end of this 8 -h infusion, $27 \pm 3 \%{ }^{3} \mathrm{H}$ and $31 \pm 5 \%{ }^{14} \mathrm{C}$ of the total dpm infused remained in the first quarter of the intestine; $11 \pm 3 \%{ }^{3} \mathrm{H}$ and $13 \pm 3 \%{ }^{14} \mathrm{C}$ dpm were in the second. The other segments had negligible amounts of radioactivity. These data are similar to those we previously reported (3). The results suggest that a significant amount of lipid remained in the mucosa as a result of L-81 treatment that would be available for transport during the unblocking phase of the experiment.

Next we sought to define the distribution of the radioactivity in the mucosa among different lipid classes at the conclusion of the initial 8-h infusion. These data are presented in Table I. As shown, $>90 \%$ of the dpm were present in the TG fraction. This indicates that the L-81 did not interfere with cellular resynthesis of the absorbed lipids. Note that the percentage of radioactivity in FFA was very low. This means that there would not be a reservoir of radiolabeled oleate available to be recycled into TG synthesis during the unblocking phase of the study.

As a final preliminary experiment, we wanted to ensure that the TO infused during the blocked period and the fatty acid infused during the unblocking portion of the study were both absorbed in the same section of the intestine. For this

Table I. Distribution of Radionuclides in Lipid Classes in Intestinal Mucosa

\begin{tabular}{ccccc}
\hline & \multicolumn{4}{c}{ Lipid class (\% of total radioactivity)* } \\
\cline { 2 - 5 } Radionuclide & $\mathrm{TG}^{\$}$ & $\mathrm{DG}^{\$}$ & $\mathrm{MG}+\mathrm{PL}^{\$}$ & FFA \\
\hline${ }^{3} \mathrm{H}$ & $92 \pm 2$ & $3.9 \pm 1$ & $3.8 \pm 1$ & \\
${ }^{14} \mathrm{C}$ & $92 \pm 1$ & $2.5 \pm 1$ & $2.2 \pm 0.6$ & $2.4 \pm 0.2$ \\
\hline
\end{tabular}

\footnotetext{
* Data are presented as mean \pm SEM.

₹ $40 \mu \mathrm{mol} / \mathrm{h}\left[{ }^{3} \mathrm{H}\right]$ glyceryl tri $\left[{ }^{14} \mathrm{C}\right]$ oleate was infused for $8 \mathrm{~h}$ and the proximal half intestine was harvested.

${ }^{\S}$ TG, triacylglycerol; DG, diacylglycerol; MG + PL, monoacylglycerol and phospholipid.
}

purpose, $\left[{ }^{3} \mathrm{H}\right]$ glyceryl trioleate plus L-81 were infused for $8 \mathrm{~h}$ and $\left[{ }^{14} \mathrm{C}\right]$ oleate (used as a model fatty acid) was only infused for the subsequent $3 \mathrm{~h}$. At the end of the experiment, the intestine was removed, cut into quarters, and radioactivity determined for each gut segment. $99 \%$ of both radionuclides were found in the proximal half of the intestine. This indicates that the fatty acid infused during the unblocking was absorbed in the same segment of intestine as the lipid that accumulated during the TO plus L-81 infusion.

Lymphatic TG output. Lymphatic TG output was measured both chemically and by reference to the specific activity of the two radionuclides infused. Because the majority of the radioactive lipid output was in the TG fraction, we took the total lymphatic radioactive lipid output in lymph to be TG. This overestimates the actual output by $3 \%$ (12). As shown in Fig. 1, the lymphatic TG output as measured by radioactivity was similar with regard to both ${ }^{14} \mathrm{C}$ and ${ }^{3} \mathrm{H}$. The lymphatic radioactive lipid output was low during the infusion of lipid plus L-81 (0-8 h) and was $\sim 5 \mu \mathrm{mol} / \mathrm{h}$ during the 7 th- 8 th $\mathrm{h}$. At the end of the 8 th $h$, the infusion of TO plus L-81 was replaced by infusion of an equivalent molar amount of linoleate. This was associated with a marked increase in the lymphatic radioactive lipid output, which reached a maximum between 11 and $12 \mathrm{~h}$ of infusion. The maximal TG output calculated from the radioactive data was $20-24 \mu \mathrm{mol} / \mathrm{h}$. From the 12 th $\mathrm{h}$ onwards, the lymphatic radioactive TG output fell progressively, presumably reflecting the depletion of the accumulated mucosal radioactive $\mathrm{TG}$.

The lymphatic TG output as determined chemically was between 8 and $10 \mu \mathrm{mol}$ during the last hour of L-81 plus triolein infusion, $60-100 \%$ higher than the output measured by radioactive $\mathrm{TG}$. This difference was probably due to a large contribution of endogenous lipid. When the L-81 infusion was stopped at the 8th h, the lymphatic TG output subsequently increased, reaching a maximum output of $\sim 53 \mu \mathrm{mol} / \mathrm{h}$. Unlike the lymphatic radioactive TG output, the lymphatic TG mass output decreased between 11 and $15 \mathrm{~h}$ and then remained relatively constant at $\sim 30 \mu \mathrm{mol} / \mathrm{h}$. This average

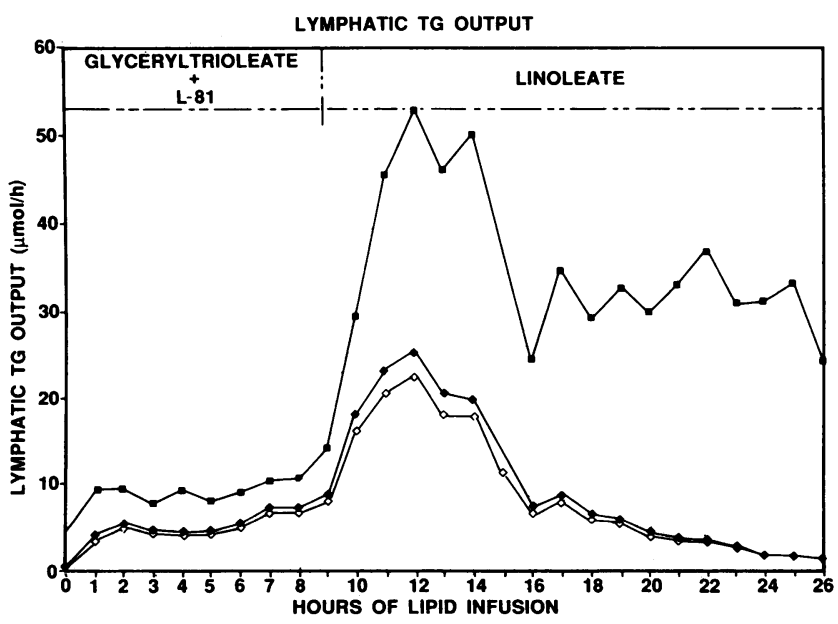

Figure 1. Lymphatic TG output after glyceryl trioleate plus L-81 infusion for $8 \mathrm{~h}$ and linoleate infusion for $18 \mathrm{~h}$. The data are the mean output from six rats. $n$, TG mass output measured quantitatively. $\bullet$, TG output as calculated from ${ }^{14} \mathrm{C}$ specific activity. $\diamond$, TG output as calculated from ${ }^{3} \mathrm{H}$ specific activity. 
value, when added to the peak TG output as determined from the radioactive data $(20-24 \mu \mathrm{mol} / \mathrm{h})$ results in the maximal TG output actually obtained by chemical measurement ( 53 $\mu \mathrm{mol} / \mathrm{h})$. These data imply that linoleate uptake and transport by the blocked enterocytes was unimpaired. It is presumed that after the supply of the monoacylglycerol derived from infused TO is exhausted (certainly after $15 \mathrm{~h}$ ), the TG transported is synthesized using sn-3-glycerophosphate as the glyceride-glycerol precursor.

The linoleate infusion did not seem to affect the unloading pattern of the lipid accumulated in the mucosa as a result of L-81 treatment. Maximal unblocking was achieved 3-4 h after the termination of the L-81 infusion, agreeing with previous studies (3). The only difference between this study and the previous ones was the fact that the maximal TG output as measured by radioactivity was diminished (1-3). This could be due to the smaller amount of lipid that was trapped in the mucosa by L- 81 infusion in the present study ( $43 \%$ of the total infused lipid, based on ${ }^{14} \mathrm{C}$ ) as compared with $55 \%$ trapped in our prior work (3). Alternatively, the discrepancy could be secondary to linoleate rather than saline being infused during the unblocking period, resulting in competition for transport of the blocked TG.

Lymph ${ }^{3} \mathrm{H} /{ }^{14} \mathrm{C}$ ratio. We infused TO that contained TO labeled in the oleoyl moiety with ${ }^{14} \mathrm{C}$ and in the glyceride-glycerol with ${ }^{3} \mathrm{H}$. We wished to determine if one of the radionuclides was preferentially lost from the lipid that accumulated in the mucosa during the blocking period and was transported during unblocking. An elevation of this ratio would have indicated hydrolysis of the esterified oleate with replacement by a nonradioactive acyl group, presumably linoleate. As shown in Fig. 2, the ${ }^{3} \mathrm{H} /{ }^{14} \mathrm{C}$ ratio remained remarkably constant during treatment with L-81, varying between 10.03 and 10.48. The ${ }^{3} \mathrm{H} /{ }^{14} \mathrm{C}$ ratio tended to be slightly higher in later hours of blocking than at the beginning, which is particularly evident during the 7 th and 8 th $h$ of infusion. During the reversal of

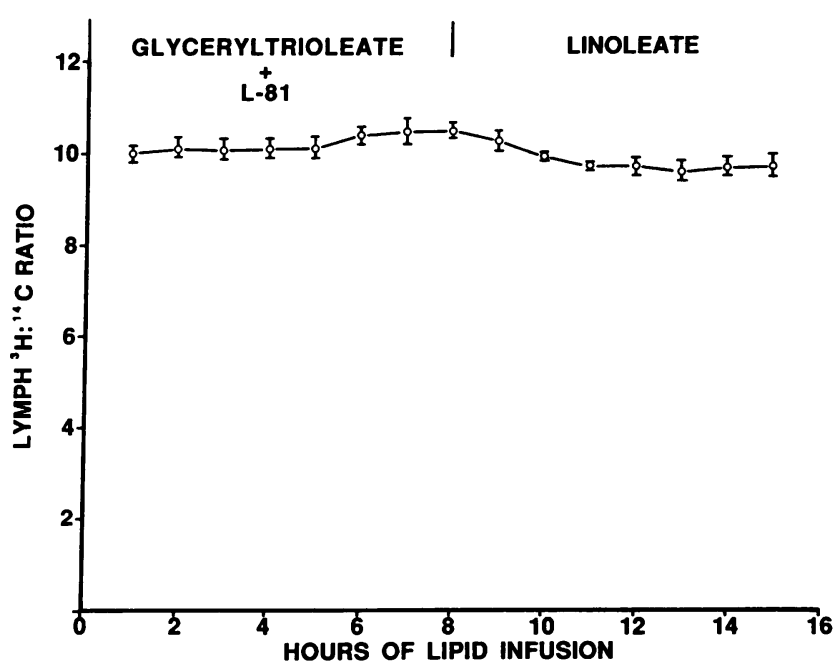

Figure $2 .{ }^{3} \mathrm{H} /{ }^{14} \mathrm{C}$ ratio in lymph neutral lipids during the hours of lipid infusion as shown on the abscissa. Glyceryltrioleate plus L-81 was infused for the first $\mathbf{8} \mathbf{h}$ as indicated and linoleate was infused subsequently. Lymph lipids were extracted (9) and treated with fluoracil (10) to remove phospholipids before analysis. The data are the mean $\pm \mathrm{SE}$ of six rats.
L-81 inhibition, the ${ }^{3} \mathrm{H} /{ }^{14} \mathrm{C}$ ratio decreased very slightly and was remarkably constant from the 9 th to 16 th $\mathrm{h}$. These data indicate two possibilities. First, the TG accumulated in the intestinal mucosa is transported out of the intestinal epithelial cells without extensive hydrolysis. Second, the TG may have undergone extensive hydrolysis, but the radioactive fatty acid released is in a restricted pool that was not diluted by the nonradioactive linoleate infused during the reversal of L-81 inhibition. This oleate was then used preferentially for reesterification. Although the former explanation is more likely, we cannot rule out the latter possibility.

Recoveries of ${ }^{14} \mathrm{C}$ and ${ }^{3} \mathrm{H}$ radioactivity. The recovery of total ${ }^{14} \mathrm{C}$ and ${ }^{3} \mathrm{H}$ radioactive lipid in lymph was $69 \pm 5$ and $63 \pm 9 \%$, respectively. These data are similar to our previous observations in which the radioactive lipid recovered from the lymph, lumen, and mucosa at the end of $8 \mathrm{~h}$ of triolein plus L-81 infusion was totaled $(1,14)$. These data thus strongly support the fact that the inhibition of lipid transport by L-81 was a specific and reversible process. Further, the addition of linoleate during reversal did not alter the delivery of blocked lipid into the lymph.

Separation of lymph TG on HPLC. To evaluate the acyl group composition of the lymph TG and the distribution of radionuclides among them, lymph TG species were separated by HPLC. A representative HPLC trace of lymph TG is shown in Fig. 3. The chromatographed lipid was collected during the peak output of TG after removal of the L-81. The figure shows that the TG transported during this period may be resolved into three major and several minor peaks based upon the acyl group composition of the TG. The TO peak is not as great as its mass would suggest (see below), which is probably due to

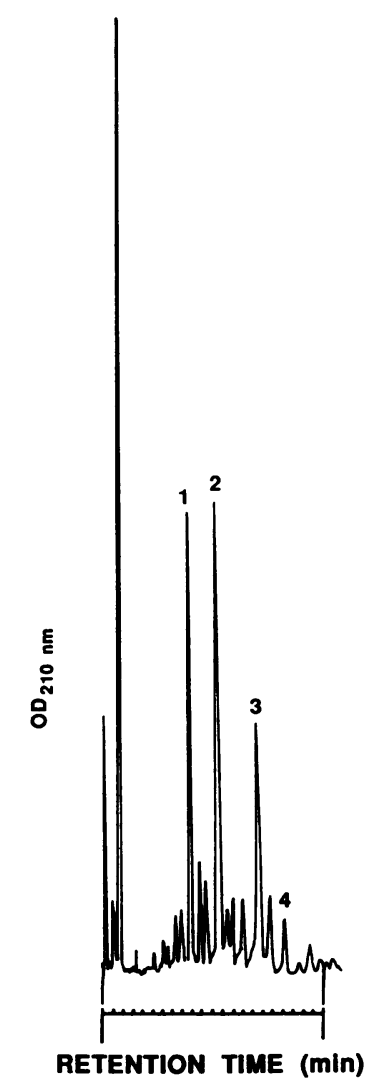

Figure 3. HPLC of a lymph sample after $12 \mathrm{~h}$ of infusion. The retention time is indicated on the abscissa. Each small, vertical division represents $2 \mathrm{~min}$. Absorbance at $210 \mathrm{~nm}$ is indicated on the ordinate. The major peaks are numbered 1-3. Peak 4, representing TO, is also shown. Each of these peaks was collected for GLC analysis. Peak 1 shows pure glyceryltrilinoleate chromatographed with retention time. Pure TO chromatographed with a retention time is indicated by peak 4 . 
the wavelength used to monitor the column output $(210 \mathrm{~mm})$. This was optimized for TL and not TO whose absorption maximum is $>228 \mathrm{~nm}$. The major peaks, 1-3, are, respectively, TL; glyceryl dilinoleate-monooleate; and glyceryl monolinoleate, dioleate. The minor peak, 4 , is TO. Substantiation of these identifications was performed by chromatographing pure standards and by analyzing the fatty acid composition of the isolated fractions by GLC.

As a first approach to identification of the peaks, we chromatographed pure TL and TO. These had retention times consistent with peak 1 and 4, respectively. It was thought that peaks 2 and 3 represented admixtures of the two TGs. Proof of these supposed structures was sought by determining the acyl group composition of each peak by GLC analysis. The results are shown in Table II. As shown, peak 1 was predominantly TL; peak 2, TG whose acyl group composition was one oleate and two linoleate molecules; peak 3, TG whose acyl group composition was two oleates and one linoleate; whereas peak 4 consisted mainly of TO. Our previous suppositions thus were confirmed.

To determine the percentage of radioactive TG applied to the column that was recovered during the HPLC procedure, known amounts of dpm (mucosal TG) were injected and samples from the column collected every $2 \mathrm{~min}$ for $46 \mathrm{~min}$. $75 \%$ of the ${ }^{3} \mathrm{H}$ and $77 \%$ of the ${ }^{14} \mathrm{C}$ dpm chromatographed were collected. These data indicate good recovery of the dpm injected. Since we could adequately separate TGs containing each of the expected major acyl group combinations and recover the majority of the lipid applied, we were in a position to observe if the mucosal TG species that accumulated in the blocked state had an altered acyl group composition when it appeared in the lymph during unblocking.

To make these observations, it was first important to know the acyl group composition of the labeled TG residing in the mucosa at the time of unblocking. Analysis of the TG extracted from the mucosa of the proximal half of the intestine at the conclusion of the blocking period demonstrated that $90 \pm 7 \%$ of the ${ }^{3} \mathrm{H}$ and $95 \pm 7 \%$ of the ${ }^{14} \mathrm{C}$ dpm had a retention time consistent with TO. Thus, at the onset of unblocking most dpm were in TO and therefore alterations in the distribution of the dpm in various TG species would indicate partial or complete hydrolysis of the TO molecule.

To examine the question of alteration in the distribution of acyl groups during unblocking, we elected to examine lymph TG at four different time points during the experiment. The first time point selected was during the blocking phase; the second, during the initial period of unblocking; the third, at the height of TG output during unblocking; and the fourth,

Table II. Fatty Acid Composition of Lymph TG Fractions 1-4 Isolated by HPLC (Mass Percent)*

\begin{tabular}{cccccccc}
\hline Fractions & $14: 0$ & $16: 0$ & $16: 1$ & $18: 0$ & $18: 1$ & $18: 2$ & $20: 4$ \\
\hline 1 & 1.5 & 2.1 & - & 0.5 & 1.3 & 93.7 & 0.6 \\
2 & 8.5 & 1.1 & 0.3 & 0.3 & 31.5 & 57.9 & 0.1 \\
3 & 6.5 & 4.4 & 0.7 & 1.5 & 58.9 & 27.5 & 0.4 \\
4 & 0.1 & 1.8 & 0.9 & 1.0 & 95.6 & 0.6 & 0.1
\end{tabular}

* The data are the mean of four lymph samples from the 12th $\mathrm{h}$ of infusion. toward the end of the unblocking phase. The results of the HPLC analyses are presented in Table III. In general, the data show that initially most dpm, both ${ }^{3} \mathrm{H}$ and ${ }^{14} \mathrm{C}$, were in $\mathrm{TO}$, but that over time this proportion diminished. At the end of the observation period, less than half of the dpm from both isotopes had retention times consistent with TO. The dpm accounted for that were not in TO were mostly in glyceryl dioleate-monolinoleate. dpm with retention times consistent with this acyl group composition grew proportionally larger over time and become equal to that found in $\mathrm{TO}$ at the end of the study. A minor number of dpm had retention times characteristic of glyceryl dilinolein-monoolein. The number of dpm in TG with this acyl group composition, indicating even more extensive degradation of the original TO, also increased over time, but was never $>20 \%$ of the total dpm. Even at the end of the observation period, no ${ }^{3} \mathrm{H}$ from glyceride glycerol appeared in the chromatogram with a retention time characteristic of TL. This is consistent with our previous speculations concerning the lack of reuse of free glycerol derived from complete hydrolysis of TG for complex lipid synthesis in the intestine (5).

Of further interest were observations on the ${ }^{3} \mathrm{H} /{ }^{14} \mathrm{C}$ ratios both in the mucosa and in lymph TG at the time points as detailed above. Although there were slight variations in the infusate, ${ }^{3} \mathrm{H} /{ }^{14} \mathrm{C}$ ratios averaged 10 . To make these various ratios comparable, each infusate was transformed to 10 . All the mucosal and lymph TG samples associated with each infusate were then corrected by the relevant number so that they too could be compared with each other.

Table IV shows the data referable to the radionuclide ratios in mucosa (see footnote) and lymph TG. It is evident that the

Table III. Percentage of dpm Appearing in Lymph TG Fractions of Specific Acyl Group Composition in Rats Receiving Linoleate during Unblocking*

\begin{tabular}{|c|c|c|c|c|}
\hline \multirow{2}{*}{$\begin{array}{l}\text { TG acyl group } \\
\text { composition }\end{array}$} & \multicolumn{4}{|c|}{$\%$ Distribution of ${ }^{3} \mathrm{H}^{*}$} \\
\hline & $6 h^{4}$ & $10 \mathrm{~h}^{4}$ & $12 \mathrm{~h}^{8}$ & $14.5 h^{8}$ \\
\hline LLL" & 0 & 0 & 0 & 0 \\
\hline LOL" & $2.0 \pm 0.5$ & $7.8 \pm 1$ & $9.8 \pm 1$ & $20 \pm 3$ \\
\hline OLO" & $12 \pm 1$ & $28 \pm 2$ & $35 \pm 1$ & $39 \pm 2$ \\
\hline OOO" & $75 \pm 3$ & $56 \pm 3$ & $41 \pm 3$ & $32 \pm 3$ \\
\hline \multirow[t]{3}{*}{ Total $^{\S}$} & 89 & 92 & 86 & 91 \\
\hline & \multicolumn{4}{|c|}{$\%$ Distribution of ${ }^{14} \mathrm{C}^{11}$} \\
\hline & $6 h^{8}$ & $10 \mathrm{~h}^{4}$ & $12 \mathrm{~h}^{8}$ & $14.5 \mathrm{~h}^{8}$ \\
\hline LLL" & 0 & 0 & 0 & 0 \\
\hline LOL" & $2.0 \pm 0.3$ & $6.2 \pm 0.9$ & $9.2 \pm 2$ & $15 \pm 3$ \\
\hline OLO" & $11 \pm 1$ & $26 \pm 1$ & $33 \pm 2$ & $39 \pm 1$ \\
\hline OOO" & $80 \pm 3$ & $57 \pm 3$ & $48 \pm 3$ & $38 \pm 3$ \\
\hline Total' & 93 & 89 & 90 & 92 \\
\hline
\end{tabular}

* Data are expressed as mean \pm SEM.

${ }^{\ddagger}\left[{ }^{3} \mathrm{H}\right]$ glyceryl $\left[{ }^{14} \mathrm{C}\right]$ oleoyl glyceryltrioleate infused for $8 \mathrm{~h}$.

$\$$ Hours of lipid infusion.

" LLL, glyceryl trilinoleate; LOL, glyceryl dilinoleate monooleate; OLO, glyceryl dioleate, monolinoleate; $\mathrm{OOO}$, glyceryl trioleate.

' Total percentage of $\mathrm{dpm}$ recovered from major chromatographic peaks. Total dpm in column effluent $=100 \%$. 
Table IV. ${ }^{3} \mathrm{H} /{ }^{14} \mathrm{C}$ Ratio in Lymph TG of Specific Acyl Group Composition in Rats Receiving Linoleate during Unblocking*

\begin{tabular}{|c|c|c|c|c|}
\hline \multirow{2}{*}{$\begin{array}{l}\text { TG acyl group } \\
\text { composition }\end{array}$} & \multicolumn{4}{|c|}{${ }^{3} \mathrm{H} /{ }^{14} \mathrm{C}$ ratio ${ }^{\ddagger}$} \\
\hline & $6 h^{8}$ & $10 \mathrm{~h}^{4}$ & $12 \mathrm{~h}^{4}$ & $14.5 \mathrm{~h}^{8}$ \\
\hline LOL" & $10 \pm 1^{\prime}$ & $12 \pm 0.4$ & $12 \pm 0.8$ & $11 \pm 0.4$ \\
\hline OLO" & $11 \pm 0.4$ & $9.1 \pm 0.3^{* *}$ & $9.1 \pm 0.3^{* *}$ & $8.4 \pm 0.3^{* *}$ \\
\hline OOO" & $8.3 \pm 0.2^{* *}$ & $7.4 \pm 0.2^{* *}$ & $7.0 \pm 0.2^{* *}$ & $7.1 \pm 0.3^{8 \$}$ \\
\hline
\end{tabular}

* Data are presented as mean \pm SEM.

${ }^{\ddagger}\left[{ }^{3} \mathrm{H}\right]$ glyceryl $\left[{ }^{14} \mathrm{C}\right]$ oleoyl glyceryl trioleate $\left({ }^{3} \mathrm{H} /{ }^{14} \mathrm{C}=10\right)$ was infused for $8 \mathrm{~h}$. Linoleate was infused subsequently.

${ }^{\S}$ Hours of lipid infusion.

"Specific acyl group composition as in Table III.

'The small amount of dpm available for determining the ratio makes this value questionable.

${ }^{* *} P<0.01$ than TG containing one more linoleate.

${ }^{\ddagger \ddagger}$ Mucosal ${ }^{3} \mathrm{H} /{ }^{14} \mathrm{C}$ ratio at $8 \mathrm{~h}$ of infusion was the same as in lymph at $6 \mathrm{~h}, 8.3 \pm 0.2$.

${ }_{\$} P<0.02$ than TG containing one more linoleate.

ratio in mucosal TO is reduced as compared with that of the infusate, indicating a small partial loss of glyceride glycerol with reacylation of nonradioactive glycerol with marked oleate. In lymph TO this trend became more marked as the duration of the experiment increased. Even at the last observation period, however, the ratio was only reduced $14 \%$ compared with the mucosa and $29 \%$ compared with the original infusate.

As the oleate in TO becomes replaced with unmarked linoleate and with conservation of the glyceride-glycerol, the ${ }^{3} \mathrm{H} /{ }^{14} \mathrm{C}$ ratio would be expected to rise. As demonstrated in Table IV, the ratio increased with each substitution. With the first acyl group exchange, the ratio increased an average of $24 \%$ and with the second, 56\%. This is close to the 33 and $66 \%$ increment that would have been expected to occur as an oleate was serially replaced with linoleate. The closeness of fit of the observed to the expected ratios substantiates the acyl group composition of the TGs at the indicated retention times. The data from LOL at the 6th $\mathrm{h}$ of infusion are not included in these considerations because the small number of $\mathrm{dpm}$ available made this result questionable.

Finally, we wished to repeat the observations made in Fig. 1 and Tables III and IV in rats who received only saline during the unblocking period rather than linoleate (group $C$ rats). Data to these points are presented in Fig. 4 and Table V. As can be seen in Fig. 4, there was a prompt rise in TG output into the lymph upon relief of the inhibition whose time course was similar to that obtained in Fig. 1. As expected, however, after peak output was reached, there was a progressive fall in lymph TG delivery to very low levels because no additional lipid was given intraduodenally. The lipid that was delivered to the lymph was predominantly TO (Table V) and remained so throughout the observation period. This contrasts with the progressive fall over time in the percentage of TG made up by TO in the linoleate-infused rats (Table III). The remainder of the radioactivity was found in TGs whose retention times were less than TO. The specific acyl group components of these various TGs were undetermined and therefore compositional percentage data are not presented.

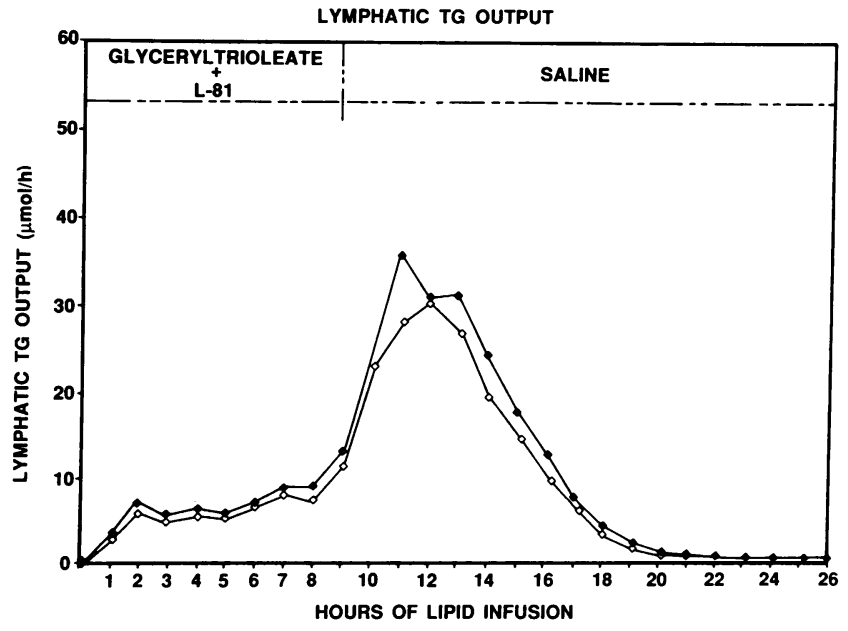

Figure 4. Lymphatic TG output after glyceryl trioleate plus L-81 infusion for $8 \mathrm{~h}$ and $0.15 \mathrm{M} \mathrm{NaCl}$ infusion for $18 \mathrm{~h}$. The data are the mean output from three rats. $\bullet$, TG output calculated from ${ }^{14} \mathrm{C}$ specific activity; $\diamond$, TG output calculated from ${ }^{3} \mathrm{H}$ specific activity.

It would also be expected that the TO delivered into the lymph in group $\mathrm{C}$ rats would have a ${ }^{3} \mathrm{H} /{ }^{14} \mathrm{C}$ ratio that was similar to that described in Table IV. Data to this point are shown in Table $\mathrm{V}$, which demonstrates the constancy of the ${ }^{3} \mathrm{H} /{ }^{14} \mathrm{C}$ ratio of TO during the entire experiment, as was seen in the group B rats. The ratios of the TGs whose retention times were less than TO had ratios that varied from 7.1 to 12 . These data are not included in the table because the acyl group composition of the TGs is unknown. An additional complicating factor is the comparatively low number of $\mathrm{dpm}$ in these fast eluting TGs, which may have yielded spurious data.

\section{Discussion}

The nonionic detergent, L-81, has been shown to effectively inhibit intestinal CM transport while simultaneously leaving intestinal VLDL transport undisturbed (15). The ability to

Table V. Percentage Distribution of dpm and ${ }^{3} \mathrm{H} /{ }^{14} \mathrm{C}$ Ratio in Lymph TO* in Rats Receiving Saline during Unblocking ${ }^{\ddagger}$

\begin{tabular}{ccccc}
\hline & \multicolumn{5}{c}{ \% distribution of radionuclide into TO } \\
\cline { 2 - 5 } Radionuclide & $6 \mathrm{~h}$ & $10 \mathrm{~h}$ & $12 \mathrm{~h}$ & $14.5 \mathrm{~h}$ \\
\hline${ }^{3} \mathrm{H}$ & $70 \pm 3$ & $71 \pm 2$ & $80 \pm 3$ & $79 \pm 2$ \\
${ }^{14} \mathrm{C}$ & $76 \pm 4$ & $74 \pm 2$ & $83 \pm 3$ & $83 \pm 2$ \\
\hline \multicolumn{5}{c}{${ }^{3} \mathrm{H} /{ }^{14} \mathrm{C}$ ratio of $\mathrm{TO}$} \\
& 6.411 \\
\hline${ }^{3} \mathrm{H}:{ }^{14} \mathrm{C}$ & $8.4 \pm 0.4$ & $7.2 \pm 0.2$ & $7.4 \pm 0.3$ & $7.4 \pm 0.3$ \\
\hline
\end{tabular}

\footnotetext{
* Glyceryltrioleate.

${ }^{\ddagger}$ Data are the mean \pm 1 SEM.

$\S 40 \mu \mathrm{mol} / \mathrm{h}\left[{ }^{3} \mathrm{H}\right]$ glyceryl $\left[{ }^{14} \mathrm{C}\right]$ oleoyl glyceryltrioleate infused for $8 \mathrm{~h}$. $0.15 \mathrm{M} \mathrm{NaCl}$ infused subsequently.

"Hours of lipid (saline) infusion.
} 
block CM transport would be expected to cause an increase in mucosal TG after lipid infusion greater than that seen in control animals. We have demonstrated this in rats $(1,2)$. Morphologic data revealed that the lipid accumulated in the endoplasmic reticulum. The blockade thus appears to be just before the Golgi apparatus. It is relieved promptly after removing the L-81 from the infusion. This situation creates an ideal circumstance in which to study the question of how the mass of TG, trapped during the blocking state, is released after the inhibition to its transport is relieved. The model is further enhanced by the fact that it simplifies the study, by analogy, of the mobilization of the large lipid droplets seen at electronmicroscopy during normal active lipid absorption (16). Under native conditions these droplets may represent part of the normal flow of TG into CM or they may represent the enterocyte TG storage pool. In the case of L-81 infusion, the situation is less complicated in that there is no $\mathrm{CM}$ production. On relieving the blockade any TG appearing in CM thus must have come from the large lipid droplets.

As a first approach to studying the transport of TG from this pool, we asked whether the stored TG was hydrolyzed in whole or in part before its delivery into the lymph. This is an important question, because if no hydrolysis is required, then how do the very large lipid droplets inside the enterocyte in the blocked state become transposed into the $\mathrm{CM}$, whose diameter is smaller and which appear in the lymph? Alternatively, if hydrolysis is required before transport, what mechanisms are involved in the hydrolytic step(s) and in moving the mass of lipid to the microsomes for reacylation?

To answer these questions, we first blocked TG transport by infusing L-81 with TO whose glyceryl and acyl portions were radiolabeled. We next demonstrated that the lipid remaining in the mucosa at the end of the blocking period was primarily labeled TO. During the unblocking step, we infused the rat with the same molar quantity of linoleate as was provided by the acyl groups of TO. We reasoned that if the mucosal TO remaining after blocking were hydrolyzed, the acyl group(s) removed would likely be replaced with a linoleate, as the enterocyte at that time would be flooded with this fatty acid. The likelihood that the fatty acid that would be reesterified would be linoleate was increased by our finding that at the end of the blocking period, only a small amount of free oleate was available for esterification (Table I).

This question was first examined by observing the ${ }^{3} \mathrm{H} /{ }^{14} \mathrm{C}$ ratio of the TG that appeared in the lymph. As shown in Fig. 2, the ratio in lymph TG was constant and did not change from that which was present in the infusate. Initially, there thus would appear to have been no hydrolysis of the TG during unblocking. Upon more careful evaluation, however, these data were found to hide some alterations in both mucosal and lymph TG. When the TO in the mucosa was isolated, it was found to have a slightly different (lower) ratio from that which was present in the infusate, clearly indicating a relative loss of $\left[{ }^{3} \mathrm{H}\right]$ glycerol. As noted (Table IV), the TO that first appeared in the lymph exactly mimicked that which was present in the mucosa. However, there were TG species present that were partial hydrolytic products of this TO. These had a higher ${ }^{3} \mathrm{H} /{ }^{14} \mathrm{C}$ ratio because of the lost $\left[{ }^{14} \mathrm{C}\right]$ oleate. Thus, only on average did the ${ }^{3} \mathrm{H} /{ }^{14} \mathrm{C}$ ratio appear to be that which was present in the original infusate.

The separation of the various TG species by HPLC allowed us to calculate the amount of lymphatic TG that had under- gone partial or complete hydrolysis. The distribution of ${ }^{3} \mathrm{H}$ among the various TG species isolated by HPLC combined with the $\left[{ }^{3} \mathrm{H}\right]$ glyceryl TO specific activity of the infusate enabled us to determine the quantity of TG transported with each acyl group composition of relevance. This revealed that half of the lipid transported into the lymph did not require hydrolysis before its egress from the enterocyte, $40 \%$ was transported after the exchange of one acyl group, and only $10 \%$ had two acyl groups that were exchanged.

These data are clearly different from similar observations in liver. In this organ, stored TG is extensively hydrolyzed and then reesterified to TG before its transport out of the hepatocyte (4). This step is blocked by chloroquine, which would suggest that the hydrolytic function is performed by lysosomal acid lipase (17). This enzyme is also present in intestinal mucosa (18) and has recently been shown to play a role in normal lipid absorption (7). In the intestine, however, chloroquine administration and thus, by inference, acid lipase did not cause a major accumulation of lipid in the mucosa and did not affect the TG mass able to be transported into the lymph. Its major role appeared to be to block the transport of TG synthesized from $s n$-3-glycerophosphate. This difference between intestine and liver in response to chloroquine might be explained on the grounds of the enzyme complement of both organs. The adult liver is only able to synthesize TG from glycerophosphate, whereas the intestine may use both this glyceride-glycerol precursor and monoacylglycerol. As applied here, the intestine's ability to synthesize TG from monoacylglycerol resulted in the capture of monoolein for lymphatic TG transport as monooleoyl, dilinoleoyl TG. We have postulated previously that the TG synthesized from monoacylglycerol is considerably more likely to be transported in CM than is the TG derived from glycerophosphate (5).

Another property of intestinal lipid metabolism that was confirmed by the present study is the rapid and efficient processing of lipids that are to be transported as CM TG. Our previous work has shown that it only takes $14 \mathrm{~min}$ for TG to appear in the lymph after its infusion into the lumen $(19,20)$ and that the fractional turnover rate of the CM TG precursor pool is very fast compared with TG in liver $(11,12)$. As shown here, the TG, blocked from transport by L-81, appears rapidly in the lymph even though the mucosa is being asked to transport TG synthesized from another FFA at the time the L-81 is removed from the infusion. The maximal output rate of blocked lipid reached on relief of the inhibition was less than the output rate when only saline was given during unblocking, as we previously demonstrated (3) or as shown here (group C rats, Fig. 4). This modest reduction in output could have been due to the reduced amount of lipid stored in the mucosa during blockade in the present study as compared with that performed previously (3) or to a true competition for transport between the TG blocked from transport by the L-81 and the linoleate infused during the unblocking part of the experiment. The present data do not allow us to distinguish between these possibilities. In this context, note that the rat can transport up to $100-110 \mu \mathrm{mol} \mathrm{TG} / \mathrm{h}$ in the lymph under ideal conditions (11). This is twice the rate reached at peak output after release of the L-81 blockade.

Note that the radiolabeled oleate does not appear in TGs whose acyl group composition is anything other than oleate and linoleate in the group B rats. Therefore, what happens to the $20 \%$ of the oleate that is hydrolyzed from the mucosal TO 
before transport? One possibility is that the oleate is transported out of the cell as FFA rather than as TG. This possibility has not been documented here but has been shown to occur under other circumstances $(21,22)$. Another possibility that cannot be excluded is that the oleate is reesterified to partial glycerides. This appears unlikely, however. For the second possibility to occur, the oleate, released during the hydrolytic process, would have to remain close to the partial glyceride from which it came. If the oleate was not nearby, a linoleate, flooding the system at this time, would be far more likely than the oleate to be used in reacylation because the hydrolytic step is likely to be at some distance from the microsomes (23), the site of reacylation.

One of the key questions that we wished to address was the extent to which the TO molecule, blocked in its exit from the enterocyte by the L-81, was hydrolyzed before its eventual transport as CM TG. This can be answered by calculating the quantity of radiolabeled TG (glyceride glycerol) whose acyl groups are composed solely or partially of oleate. Data to this point demonstrate that $80 \%$ of the oleoyl groups from mucosal TO were transported esterified to $\left[{ }^{3} \mathrm{H}\right]$ glyceride-glycerol. Only $20 \%$ of the acyl groups thus were hydrolyzed in preparation for transport as CM TG. This remarkably small amount of hydrolysis suggests that the predominant feature of the TG transported from the blocked TG pool is in direct association with the nascent $\mathrm{CM}$ during the unblocking stage. This is in direct contrast to the situation in liver and points out another dissimilarity in lipid metabolism between the two organs.

It is unknown if the findings of this study are applicable to those seen under normal lipid absorptive conditions. Certainly large lipid droplets are seen (16), much larger than would be expected for CM. These are clearly transported in some manner and thus must undergo a breakdown process. Evaluation of whether the same degree of hydrolysis of this TG occurs under normal circumstances would be hampered by the fact that CM formation and transport would occur concurrently with the transport of the lipid from the large droplets. This problem is avoided here because CM secretion is blocked until the L-81 administration is stopped.

\section{Acknowledgments}

The authors wish to acknowledge the skillful technical assistance of M. Wilson Turner and Deborah S. Drake.

This study was supported by National Institutes of Health grants HL-30553, DK-32288, DK-38760, and DK-38816 and the Research Service of the Veterans Administration. Patrick Tso is the recipient of Research Career Development Award 1 KO4 DK01575.

\section{References}

1. Tso, P., J. A. Balint, and J. B. Rodgers. 1980. Effect of hydrophobic surfactant (Pluronic L-81) on lymphatic lipid transport in the rat. Am. J. Physiol. 239:G348-G353.

2. Tso, P., J. A. Balint, M. B. Bishop, and J. B. Rodgers. 1981.
Acute inhibition of intestinal lipid transport by Pluronic L-81 in the rat. Am. J. Physiol. 241:G487-G497.

3. Tso, P., K. L. Buch, J. A. Balint, and J. B. Rodgers. 1982. Maximal lymphatic triglyceride transport rate from the small intestine. Am. J. Physiol. 242:G408-G415.

4. Mooney, R. A., and D. M. Lane. 1981. Formátion and turnover of triglyceride-rich vesicles in the chick liver cell. J. Biol. Chem. 256:11724-11733.

5. Mansbach, C. M. II, and S. Partharasarathy. 1982. A reexamination of the fate of glyceride glycerol in neutral lipid absorption and transport. J. Lipid Res. 23:1009-1019.

6. Bollman, J. L., J. C. Cain, and J. M. Grindlay. 1948. Techniques for collection of lymph from liver, small intestine or thoracic duct of the rat. J. Lab. Clin. Med. 33:1349-1352.

7. Mansbach, C. M. II, A. Arnold, and M. Garrett. 1987. Effect of chloroquine on intestine lipid metabolism. Am. J. Physiol. 253:G673G678.

8. Blanken'horn, D. H., and E. H. Ahrens. 1955. Extraction, isolation and identification of hydrolytic products of triglyceride digestion in man. J. Biol. Chem. 212:69-81.

9. Folch, J., M. Lees, and G. H. Sloane-Stanley. 1957. A simple method for the isolation and purification of total lipid from animal tissue. J. Biol. Chem. 266:497-509.

10. Börgstrom, B. 1952. Investigation on lipid separätion methods. Acta Physiol. Scand. 25:101-119.

11. Mansbach, C. M. II, and A. Arnold. 1986. Steady-state kinetic analysis of triacylglycerol delivery into mesenteric lymph. Am. J. Physiol. 251:G263-G269.

12. Mansbach, C. M. II, A. Arnold, and M. A. Cox. 1985. Factors influencing triacylglycerol delivery into mesenteric lymph. Am. J. Physiol. 249:G642-G648.

13. Morrison, W. R., and L. M. Smith. 1974. Preparation of fatty acid methyl esters and dimethylacetals. J. Lipid Res. 5:600-608.

14. Tso, P., and S. R. Gollamudi. 1984. A potent inhibitor of the transport of intestinal chylomicrons. Am. J. Physiol. 247:G32-G36.

15. Tso, P., D. S. Drake, D. D. Black, and S. M. Sabesin. 1984. Evidence for separate pathways of chylomicron and very low density lipoprotein assembly and transport by rat small intestine. Am. J. Physiol. 247:G594-G610.

16. Sabesin, S. M., and S. Frase. 1977. Electron microscopic studies of the assembly, intracellular transport, and secretion of chylomicrons by rat intestine. J. Lipid Res. 18:496-511.

17. Barrier, R. E., and P. Breacher. 1983. Hydrolysis of triolein and phospholipid vesicles and microemulsions by a purified rat liver acid lipase. J. Biol. Chem. 258:12043-12050.

18. Coats, P. M., S. A. Brown, J. Jumawan, and O. Koldovsky. 1977. Characteristics and postnatal development of the acid lipase activity of the rat small intestine. Biochem. J. 166:331-338.

19. Tso, P., V. Pitts, and D. N. Granger. 1985. Role of lymph flow in intestinal chylomicron transport. Am. J. Physiol. 249:G21-G28.

20. Tso, P., J. A. Barrowman, and D. N. Granger. 1986. Importance of interstitial matrix hydration in intestinal chylomicron transport. Am. J. Physiol. 250:G497-G500.

21. Hyun, S. A., G. V. Vahouny, and C. R. Treadwell. 1967. Portal absorption of fatty acids in lymph and portal-vein cannulated rats. Biochim. Biophys. Acta. 137:296-305.

22. McDonald, G. B., D. R. Saunders, M. Weidman, and L. Fisher. 1980. Portal venous transport of long-chain fatty acids absorbed from rat intestine. Am. J. Physiol. 239:G141-G150.

23. Mansbach, C. M. II. 1973. Complex lipid synthesis in hamster intestine. Biochim. Biophys. Acta. 296:386-402. 